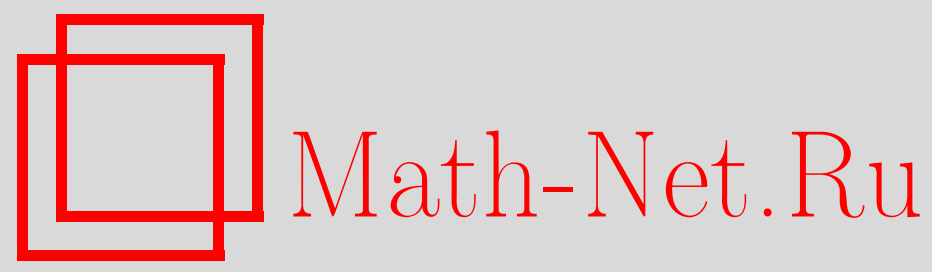

А. И. Созутов, С. И. Шахова, Строение квазислойно конечных групп, Матем. заметки, 2002, том 72, выпуск 1, 118 130

DOI: https://doi.org/10.4213/mzm409

Использование Общероссийского математического портала Math-Net.Ru подразумевает, что вы прочитали и согласны с пользовательским соглашением http://www. mathnet.ru/rus/agreement

Параметры загрузки:

IP : 54.172.240.79

26 апреля 2023 г., 12:26:18

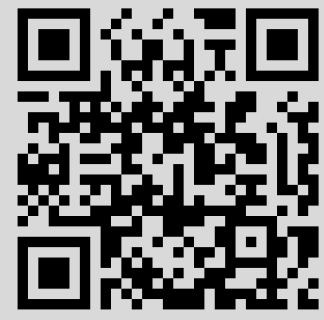




\title{
СТРОЕНИЕ КВАЗИСЛОЙНО КОНЕЧНЫХ ГРУПП
}

\author{
А. И. Созутов, С.И. Шахова
}

\begin{abstract}
Группа называется слойно конечной, если она имеет не более чем конечное число элементов каждого порядка. В статье исследовано строение бесконечных групп, все собственные подгруппы которых слойно конечны.

Библиограффия: 20 названий.
\end{abstract}

Квази- $\sigma$-группой в статье назьвается не- $\sigma$ группа, все собственные подгрупшы которой обладают свойством $\sigma$. Група назьвается слойно конечной, если она имеет не более чем конечное число элементов каждого порядка [1], [2]. Слойно конечные группы были детально изучены С. Н. Черниковым, Р. Бэром, Х. Х. Мухамеджаном, Я. Д. Половицким и др. (см., например, [3]-[5]). В данной работе заканчивается исследование строения бесконечных груп, все собственные подгрупшы которых слойно конечны, начатое в [6]-[8]. С целью полноты изложения в ней приведены формулировки всех основных результатов по квазислойно конечньг грушам из [6]-[8] (теоремы 1, 3-7).

1. Определения, основные результаты, вспомогательные предложения. В [7] доказана следующая теорема.

ТЕорема 1. Для бесконечной группы $G$, все собственные подгруппы которой слойно конечны, справедливо одно из следующих утвержсдений:

1) $G$ - слойно конечная группа;

2) $G=P \lambda\langle a\rangle$, где $P$ - черниковская полная абелева р-группа, не содержащая собственных бесконечных а-инвариантных подгрупп, $\langle a\rangle-$ примарная группа и $\left|G: C_{G}(P)\right|$ - простое число;

3) $G / Z(G)$ - простая не локально конечная группа.

Редукцию к простьм не локально конечным группам из теоремы 1 дополняет теорема существования континуального множества таких групा.

ТЕОрема 2. Пусть $\left\{G_{i}\right\}_{i \in I}$ - конечное или счетное множсество неединичных конечных или слойно конечных групп $G_{i}$ без инволюиий, $|I| \geqslant 2, n_{0}$ - достаточно большое нечетное число. Тогда свободная амальгама $X$ групп $G_{i}$ вложима в квазислойно конечную простую группу $G=G(\infty)[9]$, в которой всякая собственная подгруппа является или ииклической группой порядка, делящего $n_{0}$, или содержится в подгруппе, сопряженной с некоторой из групп $G_{i}$.

Работа выполнена при финансовой поддержке Российского фонда фундаментальных исследований, грант № 99-01-00542, и Красноярского краевого фонда науки, грант № 9 F132. 
При этом мохсно построить и такую группу $G=G(\infty)$, мультипликатор Шура которой является свободной абелевой группой счетного ранга. В этом случае для любой абелевой конечной или слойно конечной әруппы $Z$ существует квазислойно конечная группа, являюшаяся центральным расширением әруппы $G c$ помощью центральной подгруппы $Z$.

Ввиду теоремы 2 строение центра и строение факторгруппы по центру в квазислойно конечной группе могут не зависеть друг от друга. Поэтому в дальнейшем в работе будут изучаться только простые квазислойно конечные грушы. Поскольку груша, все собственные подгруппы которой конечны, также является квазислойно конечной, коснемся строения простых квазиконечных групп.

Как вытекает из известных результатов В.П. Шункова [10] (см. также [9, теорема 7.7]), простая квазиконечная группа не содержит инволюций. По теореме 35.1 из [9] любая свободная амальгама конечных групп нечетного порядка вложима в подходящую простую квазиконечную группу, в частности, множество квазиконечных групп континуально. Обсудим возможность вложений в подходящую простую квазиконечную групу амальгам конечных групп с объединенной подгруппой (вееров подгрупп). Естественные ограничения на подгрупшы вложимого веера вытекают из результатов работ [11], [6]. Почти все его подгруппы обязаны быть группами Фробениуса, неинвариантньй множитель которых совпадает с основанием [6] бесконечного веера (является объединяемой подгруппой амальгамы). Вложимость подобных амальгам в настоящее время вполне решаемая задача комбинаторной теории групп (см., например, [12], [9]).

Неизвестно, расщепляема ли произвольная простая квазиконечная группа. Более определенная ситуация с ее парами порождающих. Приведем результат из [6].

ТЕорема 3. Для любой пары неединичных әлементов $а, b$ простой квазиконечной группы $G$ найдется бесконечно много әлементов $b^{g} \operatorname{maких,~что~} G=\left\langle a, b^{g}\right\rangle$.

В частности, простая квазиконечная группа $G$ - монстр и 1-го, и 2-го рода, и 3-го рода в терминологии Шункова (определения монстров см. в вопросах 6.63, 6.64, 14.83 из Коуровской тетради [13]).

Ввиду приведенных результатов в дальнейшем в основном будут изучаться простые квазислойно конечные группы, содержащие собственные бесконечные подгрупы. Легко убедиться, что в простой квазислойно конечной групше каждая собственная подгруппа содержится в некоторой максимальной подгруппе [7]. В [7] доказаны следующие утверждения.

ТЕОрема 4. В простой бесконечной әруппе, все собственные подаруппы которой слойно конечны, любые две бесконечные максимальные подгруппы пересекаются по единичной подгруппе.

СлЕДСТВИЕ 1. Пусть $G$ - простая бесконечная әруппа, все собственные подәруппы которой слойно конечны, и Тогда $(G, H)$ - пара Фробениуса, т.е. $H \cap H^{g}=1$ для любого әлемента $g \in G \backslash H$.

Напомним, что регулярным әлементом бесконечной әруппы назьвается любой ее элемент с конечным централизатором.

ТЕОрема 5. Пусть G- простая бесконечная группа, все собственные подгруппь которой слойно конечны, $H$ - ее бесконечная максимальная подгруппа, $a$ - 
неединичный әлемент из $H$ и $X$ - непустое множество всех максимальных локально конечных подгрупп из $G$, не совпадающих с $H$ и содержащих әлемент $а$. Тогда

1) каждая подгруппа $M \in X$ конечна и является группой Фробениуса с неинвариантным мнохителем $H_{M}=M \cap H$ и ядром $F_{M}$

2) ядра $F_{M}$ и $F_{K}$ любых двух различных подгрупп $M$ и $K$ из $X$ пересекаются по единичной подгруппе;

3) для любой а-инвариантной неединичной подгруппы $F$ изядра $F_{M}$, где $M \in X$, справедливо включение $N_{G}(F) \leqslant M$;

4) все неединичные әлементы, содержашиеся в ядрах подгрупп из $X$, почти регулярны в $G$.

Неизвестно, существуют ли простые квазислойно конечные группы четного периода, т.е. содержащие инволюции. В этом смысле следующая теорема из [8] носит условный характер.

ТЕОРема 6. Если простая квазислойно конечная группа $G$ содерэсит инволюиию $i$, то $C_{G}(i)=H$-бесконечная максимальная подгруппа группь $G$, инволючия в $H$ единственна, все инволюиии в $G$ сопряжены, силовские 2-подгруппы в $G$ сопряжены и являются либо (локально) ииклическими, либо конечными (обобщенными) группами кватернионов.

Напомним, что множество $X$ (конечных) подгрупп групшы $G$ с нетривиальным пересечением $T$ назьвается веером $X$ с основанием $T$ [6]. Веер назьвается конечным или бесконечным в зависимости от конечности или бесконечности множества $X$. Произвольное подмножество $Y \subseteq X$ называется подвеером веера $X$. Бесконечньй веер $X$ с основанием $T$ называется почти правильным, если основание любого бесконечного подвеера из $X$ совпадает с $T$; бесконечньй почти правильный веер $X$ с основанием $T$ назьвается правильным, если $T \notin X$ и для любой подгрупшы $V \leqslant T$ такой, что $\left|N_{G}(V) \cap \Sigma(X)\right|<\infty$, имеет место включение $N_{G}(V) \cap \Sigma(X) \leqslant T$. В данном случае $\Sigma(X)$ - теоретико-множественное объединение подгрупп веера $X$ (его амальгама).

Строение бесконечного веера конечных подгрупп в простой квазислойно конечной группе изучено в [8]. Нам понадобится эта теорема из [8].

ТеОрема 7. Пусть $X$ - бесконечный веер всех максимальных подгрупп простой квазислойно конечной группы $G$, содержащ,х неединичный әлемент $а, u T-$ основание этого веера. Тогда справедливо одно из двух утверэсдений:

1) веер $X$ содерхит точно одну бесконечную подгруппу $H$ группь $G, T<H$ и каждая конечная подрруппа $M \in X$ есть конечная группа Фробениуса $c$ неинвариантным множителем $M \cap H$, при этом ядра любых двух конечных подгрупп веера $X$ имеют тривиальное пересечение;

2) все подгруппь веера $X$ конечны, и существует разбиение $X=Y \cup X_{1} \cup X_{2} \cup$ $\cdots \cup X_{n}$ веера $X$ на конечный или пустой веер $Y$ и конечное число $n$ правильных вееров $X_{i}$ с основаниями $T_{i}$, при этом каждая подгруппа $H \in X_{i}$ есть группа Фробениуса с неинвариантным множителем $T_{i}(i=1, \ldots, n)$ и ядра любых двух подгрупп из веера $X_{1} \cup X_{2} \cup \cdots \cup X_{n}$ пересекаются тривиально.

Теорема 7 дополняется в настоящей работе следующим результатом. 
Теорема 8. Если бесконечная простая квазислойно конечная группа $G$ содержит пару конечных максимальных подгрупп с нетривиальным пересечением $T$, не являюшихся группами Фробениуса, то $G$ есть подгруппа группь автоморфизмов бесконечного однородного локально конечного графа, транзитивно действующая на мнохсестве его вериин.

Наши исследования завершают результаты о парах порождающих простых квазислойно конечных груп, которые с помощью теоремы 1 легко экстраполировать на произвольные не черниковские квазислойно конечные группы.

ТЕорема 9. Для любой пары неединичных әлементов а, b бесконечной простой квазислойно конечной группы $G$, хотя бы один из которых не является инволюиией, найдется бесконечно много әлементов $b^{g}$ таких, что $G=\left\langle a, b^{g}\right\rangle$.

СлЕдствиЕ 2. Бесконечная простая квазислойно конечная группа является монстром 1-го, 2-го и 3-го рода. В частности, для квазислойно конечных әрупп полохительно решаются вопросы 13.53 и 14.83 из Коуровской тетради [13].

ДокАЗАТЕЛЬСтво. Следствие вытекает непосредственно из теоремы 9, формулировок вопросов 13.53, 14.83 и определений монстров (см. вопросы 6.63, 6.64, 14.83 из Коуровской тетради [13]).

Напомним, что выражение “почти для всех" означает “для всех, кроме, быть может, конечного числа". Следующая теорема усиливает утверждение теоремы 9 для некоторых пар порождающих простой квазислойно конечной групшы.

ТЕОрема 10. Пусть $G$ - бесконечная простая группа, все собственные подзруппы которой слойно конечны, $a, b$ - ее неединичные әлементы $и H \geqslant C_{G}(a)$, $M \geqslant C_{G}(b)$ - максимальные подгруппы в $G$. Тогда

1) если $|H|=\infty,|M|=\infty$ и $M \notin H^{G}$, mо $G=\langle a, c\rangle$ для каждого $c \in b^{G}$;

2) если $|H|<\infty,|M|=\infty$, то $G=\langle a, c\rangle$ почти для всех $c \in b^{G}$;

3) если $|a|=|b|-$ простое число и подгруппь $\langle a\rangle,\langle b\rangle$ не сопряжены в $G$, то $G=\langle a, c\rangle$ почти для всех $c \in b^{G}$

4) если $|H|<\infty,|a|,|b|-$ различные простые числа и $C_{G}(a)$ не содерэит әлементов из $b^{G}$, то $G=\langle a, c\rangle$ почти для всех $c \in b^{G}$;

5) если $|a|=2,|b| \neq 2 u b^{a}=b^{-1}$, то $G=\langle b, c\rangle$ почти для всех элементов $c \in G$, инвертируемых инволюиией $а$.

Нам понадобятся несколько известных результатов, в основном признаков непростоты групп с некоторыми заданными системами конечных фробениусовых подгрупп. На них мы будем ссылаться как на предложения с соответствующими номерами.

Следующее предложение легко вытекает из свойств конечных групп Фробениуса (см., например, [14], [15]).

ПРЕДЛОЖЕНИЕ 1. Пусть $L$ - конечная группа Фробениуса с ядром $F$ инеинвариантным множителем (дополнением) Н. Тогда

1) порядки ядра $F$ и дополнения $H$ взаимно просты;

2) если $1 \neq K<L u(L, K)$ - пара Фробениуса, то $K \in H^{L}$;

3) $F$ - нильпотентная группа ступени $\leqslant f(|H|)$;

4) если $H$ содержит инволючию $i$, то она единственна в $H, F$ - абелева группа и $і$ инвертирует $F$; 
5) если порядок Н нечетен, то все әлементы простых порядков из Н порохдают в ней ииклическую подгруппу;

6) если $L=\langle a, b\rangle, a, b \in L \backslash F, u$ порядки әлементов а и $b$ - простые числа, то либо $H$ - ииклическая группа, либо $H \simeq S L_{2}(3)$, либо $H \simeq S L_{2}(5)$;

7) если $L=\langle a, b\rangle$ u $b \in F$, то $L=\left\langle a, a^{b}\right\rangle$ и подгруппы $H u\langle a\rangle$ сопряжсены в $L$.

Частньм случаем основной теоремы из [16] является

ПРЕДЛОЖЕНИЕ 2. Пусть $(G, H)$ - пара Фробениуса, $a \in H$ - элемент простого порядка $p>2$ и все подгруппь $\left\langle a, a^{g}\right\rangle$, где $g \in G \backslash H$, конечны. Тогда $G=F \lambda H, \boldsymbol{s}$ частности, $G$ не проста.

ПРЕДЛОЖЕНИЕ 3 (основная теорема из [17]). Пусть $G-$ бесконечная групnа, $H-$ ее собственная подгруппа, $a$ - ее элемент простого порядка $p>2$ и почти для всех әлементов $a^{g}$, где $g \in G \backslash H$, подгруппа $\left\langle a, a^{g}\right\rangle$ является конечной группой Фробениуса с неинвариантным множителем $\langle a\rangle$. Тогда либо $\left|a^{G}\right|<\infty$, либо $G=F \lambda N_{G}(\langle a\rangle)$ и $\left.F\right\rangle\langle a\rangle$ - әруппа Фробениуса с неинвариантным множителем $\langle a\rangle$.

ПРЕДЛОЖЕНИЕ 4 [18, теорема 3]. Пусть $(G, H)$ - пара Фробениуса, $i \in H-$ - инолючия и для некоторого неединичного әлемента $a \in G$ конечного порядка, большего 2, и всех әлементов $g \in G \backslash H$ подгруппь $\left\langle i, a^{g}\right\rangle$ конечны. Тогда $H=C_{G}(i)$, $i$ - единственная инволюиия в $H$ и или $G=F \lambda H$, где $F$ - периодическая абелева подгруппа без инволюиий, или $\left\langle a^{G}\right\rangle$ - периодическая абелева подгруппа без инволюиий.

ПРЕДЛОЖЕНИЕ $5[18$, теорема 4]. Пусть $(G, H)$ - пара Фробениуса, $H$ - локально конечная подгруппа, $a \in H, b \in G$ - әлементы простых порядков $p, q$ соответственно, $p q>4$, и выполняются следуюшие условия:

1) для любого әлемента $g \in G$ подгруппа $L_{g}=\left\langle a, b^{g}\right\rangle$ конечна;

2) ядра любых двух различных максимальных локально конечных фробениусовых подгрупп из $G$ с дополнением $\langle a\rangle$ пересекаются по единичной подгруппе. Тогда либо $b^{G} \cap H=\varnothing u\langle a\rangle \cap\left\langle b^{G}\right\rangle=1$, либо с точностью до обозначения $b \in H$ объединение ядер всех фробениусовых подгрупп группы $G$ с неинвариантным множителем $\langle a\rangle$ есть нормальная подгруппа $F$ группь $G u G=F 入 H$.

Следующее предложение вьводится непосредственно из теоремы 7 (см. также [8, теорема 2]).

ПРЕДЛОЖЕНИЕ 6. Пусть $X$ - бесконечный веер конечных подгрупп простой квазислойно конечной группь $G$, содержащих неединичный почти регулярный әлемент а. Тогда существует разбиение $X=Y \cup X_{1} \cup X_{2} \cup \cdots \cup X_{n}$ веера $X$ на конечный или пустой веер $Y$ и конечное число $n$ правильных вееров $X_{i}$ с основаниями $T_{i}$. При этом каждая подгруппа $H \in X_{i}$ есть группа Фробениуса с неинвариантным множителем $T_{i}(i=1, \ldots, n)$.

\section{2. Доказательство теорем $2,8$.}

ДоКАЗАТЕЛЬСТВо ТЕОРЕМЫ 2. Итак, пусть $\left\{G_{i}\right\}_{i \in I}-$ конечное или счетное множество неединичных конечных или слойно конечных групп $G_{i}$ без инволюций, $|I| \geqslant 2$, $n_{0}$ - достаточно большое нечетное число и $X-$ свободная амальгама групп $G_{i}$. 
Ввиду теоремы 35.1 из [9] амальгама $X$ вложима в квазислойно конечную простую группу $G=G(\infty)[9]$, в которой всякая собственная подгруппа является или циклической группой порядка, делящего $n_{0}$, или содержится в подгрупе, сопряженной с некоторой из групп $G_{i}$. Кроме того, как утверждается в доказательстве теоремы 35.1 из [9], существует такая група $G=G(\infty)$, копредставление которой можно построить по правилу из $[9$, п. 34.3, с. 346-348], ввиду [9, лемма 34.14$]$ удовлетворяющему условию $R$ для копредставлений в свободном произведении $F$ груп $G_{i}$. Поэтому в силу леммы 34.2 и следствия 25.1 из [9] градуированное копредставление данной групшы $G=F / N$ aсферично. По теореме 31.1 из [9] в этом случае мультипликатор Шура групшы $G$ - группа $N /[N, F]$ - является свободной абелевой группой счетного ранга. Пусть $Z$ - произвольная конечная или слойно конечная абелева группа. В силу изложенного вьше существует гомоморфизм группы $N /[N, F]$ на группу $Z[19]$. Пусть $M-$ полньй прообраз в $N$ ядра этого гомоморфизма. Очевидно, что подгрупша $M$ нормальна в $F, N / M=Z(F / M)$, $N / M \simeq Z$ и $(F / M) /(N / M) \simeq G$. Теорема 2 доказана.

ДокАЗАТЕЛЬСТво ТЕОРЕМЫ 8 . Пусть $G$ - бесконечная простая квазислойно конечная группа, $H$ и $M$ - ее максимальные конечные подгруппы, имеющие нетривиальное пересечение $T$ и не являющиеся групами $\Phi$ робениуса. Поскольку $M$ не група $\Phi$ робениуса, то в $M \backslash H$ найдется элемент $x$ такой, что пересечение $T^{x} \cap T \neq 1$. Ввиду максимальности $H$ в $G N_{G}(H)=H$ и $H^{x} \neq H$. Так как $H \cap H^{x} \neq 1$, то в качестве $M$ можно взять подгруппу $H^{x}$. Таким образом, считаем, что $M$ сопряжена с $H$. Построим на множестве $X=\left\{H^{g} \mid g \in G\right\}$ граф $\Gamma$, соединяя ребрами те подгруппы из $X$, которые имеют нетривиальное пересечение. Граф Г мы называем сетью. Понятно, что $G$ изоморфно вложима в $\operatorname{Aut}(\Gamma)$ в силу естественного действия сопряжением. Также очевидно, что $G$ действует на $Г$ транзитивно.

Докажем, что сеть Г является регулярным локально конечньм графом. Рассмотрим множество $S$ подгрупш $H^{g}$, где $g \in G$, каждая из которых не совпадает с $H$, но имеет с ней нетривиальное пересечение. Допустим, что множество $S$ бесконечно. В силу конечности подгруппы $H$ найдется такой элемент $h \in H$, которьй содержится в бесконечном множестве $Y$ подгрупп из $S$. Но в этом случае $Y$ - бесконечный веер подгрупп вида $H^{g}$. Ввиду теоремы 7 и предложения 6 почти все подгрупшы такого веера должны быть группами Фробениуса. Получили противоречие с выбором подгрупшы $H$. Следовательно, в $S$ входит лиш конечное число подгрупп $H^{g}, g \in G$, и в силу того, что группа $G$ действует транзитивно на $X, \Gamma$ является локально конечным регулярным графом степени $|S|<\infty[20]$.

Покажем, что графф $Г$ связен и бесконечен. Пусть $\Gamma_{H}-$ связная компонента граффа $\Gamma$, содержащая подгруппу $H$. Очевидно, что группа $G$ как группа подстановок множества $\Gamma$ действует импримитивно на $Г$ и $\Gamma_{H}$ - один из блоков импримитивности группы $G$. Поскольку для каждого элемента $h \in H$ имеем $H^{h}=H$, то $h \in N_{G}\left(\Gamma_{H}\right)$ и $H \subseteq N_{G}\left(\Gamma_{H}\right)$. Аналогично $H^{x} \subseteq N_{G}\left(\Gamma_{H}\right)$ для любой подгрупшы $H^{x} \in S$. Ввиду максимальности $H$ и $H^{x}$ в $G\left\langle H, H^{x}\right\rangle=G$. А значит, $N_{G}\left(\Gamma_{H}\right)=G, \Gamma=\Gamma_{H}$ и $Г$ является связным и, очевидно, бесконечньг графом. Теорема доказана.

3. Доказательство теорем 9, 10. Нам понадобятся несколько достаточных условий бесконечности централизатора неединичного элемента $a$ в простой квазислойно-конечной групше $G$.

ЛЕмма 1. Пусть $G$ - простая квазислойно конечная группа и а-ее неединичный әлемент. При выполнении хотя бы одного из следующих нижсе условий 
централизатор $C_{G}(a)$ бесконечен.

1) Элемент а содержится в собственной бесконечной подгруппе группы $G$.

2) Элемент а перестановочен с неединичным әлементом из $G$, иентрализатор которого бесконечен в $G$.

3) Элемент а перестановочен с инволючией из $G$.

ДокАЗАТЕЛЬСТво. 1) Элемент $a$ содержится в собственной бесконечной подгруппе $H$ групшы $G$, которая слойно конечна по условию. Следовательно, $\left|H: C_{H}(a)\right|<\infty$, $\left|C_{H}(a)\right|=\infty$ и $\left|C_{G}(a)\right|=\infty$.

2) Пусть $1 \neq b \in G, a b=b a$ и $\left|C_{G}(b)\right|=\infty$. Тогда $a \in C_{G}(b)$ и $\left|C_{G}(a)\right|=\infty$ по утверждению 1) леммы.

3) По теореме 6 централизатор каждой инволюции из $G$ бесконечен и $\left|C_{G}(a)\right|=\infty$ по утверждению 2) леммы. Лемма доказана.

ЛЕмма 2. Если неединичный әлемент а группы $G$ содержится в бесконечном множестве $X$ ее конечных нильпотентных групп, то его иентрализатор $C_{G}(a)$ бесконечен.

ДоКАЗАТЕЛЬСтво. Если множество различных центров $Z(H)$ подгрупп $H \in X$ бесконечно, то $C_{G}(a)$ - бесконечная подгруппа ввиду очевидных включений $Z(H) \leqslant C_{G}(a)$.

Если множество центров $Z(H)$, где $H \in X$, конечно, то среди них найдется центр $Z$, совпадающий с центрами $Z(H)$ для подгруп $H$ бесконечного подмножества $Y \subseteq X$. В этом случае бесконечна подгруппа $T=C_{G}(Z)$. Поскольку $a \in T$, то $C_{G}(a)$ бесконечен по лемме 1. Лемма доказана.

Для произвольного неединичного элемента $x \in G$ обозначим через $N_{x}$ объединение ядер всех конечных фробениусовьг подгрупп из $G$, неинвариантный множитель которых совпадает с $\langle x\rangle$.

Лемма 3. Пусть әлемент а почти регулярен в $G$ и $1 \neq b \in N_{a}$. Тогда имеют место неравенства $\left|C_{G}(b)\right|<\infty u\left|N_{a} \cap b^{G}\right|<\infty$.

ДокАЗАТЕЛЬСТво. Предположим, что $\left|C_{G}(b)\right|=\infty$ и $H$ - максимальная подгруппа из $G$, содержащая $C_{G}(b)$ (ее существование доказано в $[7$, лемма 1.3$\left.]\right)$. По условиям леммы $L=\langle a, b\rangle$ - группа $\Phi$ робениуса с ядром, содержащим элемент $b$. Ввиду регулярности элемента $a$ и утверждения 1$)$ леммы $1 a \notin H$. Но тогда по теореме $5 L-$ группа Фробениуса с неинвариантным множителем $L \cap H$, содержащим элемент $b$, что противоречит предложению 1. Следовательно, $\left|C_{G}(b)\right|<\infty$.

Докажем второе неравенство. Предположим, что бесконечно много подгрупп $L_{g}=$ $\left\langle a, b^{g}\right\rangle$, где $g$ пробегает некоторое бесконечное подмножество $Y \subseteq G$, являются конечными группами Фробениуса и содержат элемент $b^{g}$ в своем ядре $F_{g}$. Тогда элемент $b$ содержится в пересечении ядер $g F_{g} g^{-1}$ групп $\Phi$ робениуса $g L_{g} g^{-1}$ с неинвариантньми множителями $\left\langle g a g^{-1}\right\rangle$, где $g \in Y$. Если множество ядер $g F_{g} g^{-1}$, где $g \in Y$, бесконечно, то централизатор $C_{G}(b)$ бесконечен по лемме 2. Однако это противоречит доказанному неравенству $\left|C_{G}(b)\right|<\infty$. Следовательно, множество указанных ядер конечно. Тогда для элементов $g$ некоторого бесконечного подмножества $Z \subseteq Y$ все ядра $g F_{g} g^{-1}$ совпадают и равны некоторой подгруппе $F$. В этом случае $g a g^{-1} \in N_{G}(F)$ для каждого $g \in Z$ и ввиду бесконечности $Z$ и конечности $C_{G}(a)$ заключаем, что подгруппа $N_{G}(F)$ бесконечна. Но тогда и подгруппа $C_{G}(b)$ бесконечна (лемма 1 ), снова противоречие. Следовательно, $\left|N_{a} \cap b^{G}\right|<\infty$ и лемма доказана. 
ДоКАЗАТЕЛЬСТВо ТЕОРЕмЫ 9. Предположим, что теорема 9 неверна, т.е. в некоторой простой квазислойно конечной групе $G$ найдутся неединичные элементы $a$ и $b$ c $|a| \cdot|b|>4$ такие, что почти для всех элементов $c \in b^{G}$ подгрупшы $\langle a, c\rangle$ конечны. Леммы 4-11 будут доказываться при этом предположении.

Лемма 4. $C_{G}(a)$ - конечная подгруппа, порядок әлемента а нечетен и мохно считать, что $|a|=p$ - простое число.

ДокАЗАТЕЛЬСтво. Предположим, что подгруппа $C_{G}(a)$ бесконечна. По лемме 1.3 из [7] подгруппа $C_{G}(a)$ содержится в некоторой максимальной подгруппе $H$ групшы $G$. Пусть $X$ - множество элементов $c \in b^{G}$, для которых подгруппа $\langle a, c\rangle$ бесконечна. Понятно, что $X$ - конечное множество и для любого элемента $x \in X$ вьполняется $\langle a, x\rangle=G$ и $x \notin H$. Также очевидно, что $C_{G}(a) \leqslant N_{G}(X)$. По условиям $X$ - конечное множество, а подгруппа $C_{G}(a)$ бесконечна. Отсюда заключаем, что индекс подгрупшы $C_{G}(a) \cap C_{G}(x)$ в $C_{G}(a)$ конечен и $H \cap H^{x} \neq 1$ при $x \in X$. Однако по следствию $1(G, H)$ - пара Фробениуса. Полученное противоречие означает, что $X$ - пустое множество и, значит, в $G$ конечны все подгрупшы вида $\left\langle a, b^{g}\right\rangle$, где $g \in G$.

Так как для любых неединичных элементов $r \in\langle a\rangle, s \in\langle b\rangle$ все подгруппы $\left\langle r, s^{g}\right\rangle$ также конечны, то для доказательства теоремы и в том числе данной леммы достаточно ограничиться рассмотрением следуюших случаев:

1) $|a|=2,|b| \geqslant 3$

2) $|a| \geqslant 3,|b|=2$;

3) $|a|=|b|=p, p$-нечетное простое число;

4) $|a|=p,|b|=q$, где $p$ и $q$ - различные простые числа.

По следствию $1(G, H)$ - пара Фробениуса. Ввиду простоты групшы $G$ и предложения 4 первый из перечисленных случаев невозможен.

Ввиду доказанной вьше конечности всех подгрупп вида $\left\langle a, b^{g}\right\rangle$ все подгрупшы вида $\left\langle b, a^{g}\right\rangle$ также конечны. Поэтому второй случай с учетом теоремы 6 , следствия 1 и предложения 4 невозможен аналогично первому.

Пусть $|a|=|b|=p, p$ - нечетное простое число. Ввиду бесконечности максимальной подгруппы $H \geqslant C_{G}(a)$ и теоремы 5 для любого элемента $b^{g} \in G \backslash H(g \in G)$ $L_{g}=\left\langle a, b^{g}\right\rangle-$ конечная группа Фробениуса. По предложению 1 подгруппы $\langle a\rangle,\left\langle b^{g}\right\rangle$ сопряжены в $L_{g}$ и без ограничения общности можно считать, что $a=b$. Поскольку $(G, H)$ - пара $\Phi$ робениуса, то для групшы $G$, ее собственной подгруппы $H$ и элемента $a$ выполняются все условия предложения 2 . По предложению $2 G$ не проста. Следовательно, рассматриваемьй случай не может иметь места.

Пусть, наконец, $|a|=p,|b|=q$, где $p$ и $q-$ различные простые числа, которые ввиду рассмотренных ранее случаев 1$), 2$ ) можно считать отличными от 2 . Снова по следствию $1(G, H)$ - пара Фробениуса и, применяя теорему 5 к паре $(G, H)$ и подгруппам $L_{g}=\left\langle a, b^{g}\right\rangle\left(b^{g} \in b^{G} \backslash H\right)$, которые все конечны по доказанному выше, убеждаемся, что выполняются все условия предложения 5 . По предложению $5 G$ не проста, окончательное противоречие. Следовательно, централизатор $C_{G}(a)$ конечен.

Ввиду леммы $1|a|$ - нечетное число, которое можно считать простьм ввиду того, что если теорема верна для простых $|a|$, то она тем более справедлива и для составных значений $|a|$. Лемма доказана.

Лемма 5. $C_{G}(b)$ - конечная подгруппа и мохнно считать, что $|b|=q$-нечетное простое число. 
ДокАЗАТЕЛЬСТво. Как уже отмечалось, если теорема верна для простых $|b|$, то она тем более справедлива и для составных значений $|b|$. Поэтому с учетом рассмотренного в доказательстве леммы 4 случая $|b|=2$ в дальнейшем будем считать, что $|b|=q-$ простое нечетное число. Итак, $|a|=p$ (лемма 4 ), $|b|=q$, где $p$ и $q$ - необязательно различные нечетные простые числа.

Предположим, что $\left|C_{G}(b)\right|=\infty$, и пусть $M$ - максимальная подгруппа, содержащая $C_{G}(b)$, существование которой гарантировано леммой 1.3 из [8]. Рассмотрим веер $X$ всех максимальных подгрупп группы $G$, содержащих элемент $a$. Ввиду конечности $C_{G}(a)$ и леммы $1 X$ состоит из конечных подгрупп. Поскольку класс $b^{G}$, очевидно, бесконечен и почти весь содержится в амальгаме $\Sigma(X)$ веера $X$, то веер $X$ бесконечен. По теореме $7 X$ состоит из конечных фробениусовых подгрупп $L$ с неинвариантными множителями $H_{L}$, содержашими элемент $a$. Пусть $b^{g} \in L$. По теореме $5 L$ - группа $\Phi$ робениуса с дополнением $L \cap M^{g}$ и по предложению $1 H_{L}=\left(L \cap M^{g}\right)^{x}$ для некоторого элемента $x \in L$. Но тогда $a \in M^{g x}$ и $C_{G}(a)$ бесконечен по лемме 1 , что противоречит лемме 4. Следовательно, $\left|C_{G}(b)\right|<\infty$. Лемма доказана.

ЛЕмма 6. Почти для всех әлементов вида $b^{g}$ подгруппы $M_{g}=\left\langle a^{g}, b\right\rangle$ конечны.

ДОКАЗАТЕЛЬСТВО. В силу свойств групшы $G$ подгруппы $M_{g}$ могут быть либо конечными, либо совпадать с $G$. Если для некоторого элемента $g \in G$ имеет место равенство $M_{g}=\left\langle b, a^{g}\right\rangle=G$, то $G=\left\langle b, a^{g}\right\rangle^{g^{-1}}=\left\langle a, b^{g^{-1}}\right\rangle=L_{g^{-1}}$. Ввиду конечности $C_{G}(b)$ (лемма 5) и предположения о конечности подгрупп $L=\left\langle a, b^{x}\right\rangle$ почти для всех элементов $b^{x} \in b^{G}$ равенство $G=L_{g^{-1}}$ может выполняться лишь для конечного множества $Y$ элементов $g \in G$. Следовательно, для $g \in G \backslash Y$ выполняется $M_{g} \neq G$, т.е. почти для всех элементов $a^{g} \in a^{G}$ подгрупшы $M_{g}=\left\langle b, a^{g}\right\rangle$ конечны. Лемма доказана.

ЛЕмма 7. В случае $p=q$ теорема верна.

ДокАЗАТЕЛЬСтво. Пусть $p=q$. Рассмотрим веер $X_{a}$ всех конечных подгрупп $L_{g}=$ $\left\langle a, b^{g}\right\rangle, g \in G$. В силу принятых предположений веер $X_{a}$ бесконечен. Ввиду теоремы 7 и предложения 6 почти все подгрупшы $L_{g}=\left\langle a, a^{g}\right\rangle \in X_{a}$ являются конечньми группами Фробениуса с дополнениями $H_{g}$, содержащими элемент $a$. Если бы дополнение $H_{g}$ в $L_{g}$ имело четньй порядок, то ввиду единственности инволюции в $H_{g}$ (предложение 1$)$, теоремы 6 и леммы 1 мы получили бы $\left|C_{G}(a)\right|=\infty$, вопреки лемме 4. Следовательно, дополнения $H_{g}$ фробениусовых подгрупп $L_{g} \in X_{a}$ имеют нечетньй порядок. Ввиду их порожденности двумя элементами одного и того же простого порядка $p$ заключаем, что $H_{g}=\langle a\rangle$ и подгрупшы $\langle a\rangle,\left\langle b^{g}\right\rangle$ сопряжены в $L_{g}$ (предложение 1 ). Отсюда заключаем, что почти для каждого элемента $a^{g}$, где $g \in G, L_{g}=\left\langle a, a^{g}\right\rangle$ есть конечная группа $\Phi$ робениуса с неинвариантньм множителем $\langle a\rangle$. По предложению $3 G=F \lambda N_{G}(\langle a\rangle)$. Однако это противоречит простоте групшы $G$. Следовательно, при $|a|=|b|$ теорема верна и лемма доказана.

Итак, далее считаем, что $|a|=p \neq q=|b|$. Напомним, что $N_{a}\left(N_{b}\right)$ - множество всех элементов из ядер конечных груп $\Phi$ робениуса из $G$ с неинвариантным множителем $\langle a\rangle(\langle b\rangle)$.

ЛЕмма 8. Почти для каждого әлемента $b^{g} \in b^{G}$ подгруппа $L_{g}=\left\langle a, b^{g}\right\rangle$ является группой Фробениуса с ииклическим дополнением порядка $p q$, мноэества $N_{a}$ $u N_{b}$ бесконечны, имохно считать, что $а b=b a$. 
ДокаЗАтельство. Рассмотрим веер $X_{a}$ конечных подгрупш $L_{g}=\left\langle a, b^{g}\right\rangle$, где $g \in G$. В силу бесконечности класса $b^{G}$ веер $X_{a}$ бесконечен. Ввиду леммы 4 и предложения 6 почти каждая подгруппа $L_{g}$ из $X_{a}$ есть группа $\Phi$ робениуса с дополнением $H_{g}$, содержащим элемент $a$. По лемме 3 элемент $b^{g}$ не содержится в ядрах почти всех подгрупा $L_{g}$. Из предложения 1 и лемм 1, 4 следует, что неинвариантньй множитель каждой из фробениусовых подгрупп $L_{g}$ имеет нечетньй порядок. Снова применяя предложение 1 , заключаем, что почти для всех $g \in G$ неинвариантные множители $H_{g}$ фробениусовых подгрупा $L_{g} \in X_{a}$ - циклические группы порядка $p q$. В частности, элемент $a$ перестановочен с некоторым элементом из $H_{g} \cap b^{G}$, и без ограничения общности можно считать, что $a b=b a$.

Далее, ввиду теоремы 7 ядра $F_{g}$ фробениусовых подгрупп $L_{g}$ содержатся в ядрах соответствующих максимальных конечных фробениусовых подгрупп, которые попарно пересекаются по единичной подгрупп, а множество их возможных дополнений, содержаших элемент $a$, конечно. Отсюда в силу бесконечности веера $X_{a}$ заключаем, что множество различньх ядер $F_{g}$ фробениусовых подгрупп $L_{g} \in X_{a}$ бесконечно и поэтому $N_{a}$ - бесконечное множество. Этот факт можно получить и другим путем. Если множество $N_{b}$ конечно, то и число различных ядер $F_{g}$, где $L_{g} \in X_{a}$, конечно. Но тогда для некоторого ядра $F=F_{g}$ пересечение $N_{G}(F) \cap b^{G}$ бесконечно и по утверждению 1$)$ леммы $1 C_{G}(b)$ - бесконечная группа, что противоречит лемме 5.

Далее, ввиду леммы 6 заключаем, что рассматриваемая ситуация симметрична относительно элементов $a$ и $b$. Поэтому и $N_{b}$ - бесконечное множество. Лемма доказана.

ЛЕмма 9. Имеет место неравенство $\left|N_{a} \backslash N_{b}\right|<\infty$.

ДокАЗАТЕльСтво. Ввиду свойств конечных групп Фробениуса [14], [15] элемент вида $b c$, где $c \in N_{b}$, сопряжен с элементом $b$ в подгруппе $\langle b, c\rangle$, и в силу леммы 8 только для конечного числа элементов $c \in N_{b}$ подгруппы

$$
L^{+}=\langle a, b c\rangle, \quad L^{-}=\left\langle a, b^{-1} c\right\rangle
$$

не являются группами Фробениуса с циклическим неинвариантным множителем порядка $p q$. Поскольку конечное число таких "нехороших" элементов не влияет на утверждение доказьваемой леммы, то без ограничения общности мы можем считать, что $N_{b}$ уже не содержит таких элементов.

По лемме $8 b \in C_{G}(a)$ и, значит, $a^{c} \in L^{+} \cap L^{-}$. Из предложения 1 следует, что подгрупшы $\langle a\rangle$ и $\left\langle a^{c}\right\rangle$ сопряжены в $L^{+}$и $L^{-}$с помощью некоторого элемента $c_{a} \in N_{a}$, при этом в силу утверждения 6) предложения 1 элемент $c_{a}$ принадлежит пересечению ядер фробениусовых подгрупп $L^{+}$и $L^{-}$. Отсюда также выводим, что $c=r c_{a}$, где $r \in N_{G}(\langle a\rangle)$.

Пусть $Z$ - веер всех максимальных подгрупп из $G$, содержащих элемент $a$. Ввиду лемм $4,1 Z$ состоит только из конечных подгруп. Так как амальгама $\Sigma(Z)$ веера $Z$ почти полностью содержит бесконечньй класс $b^{G}$, то веер $Z$ бесконечен. По теореме 7 получаем разбиение $Z=Z_{1} \cup Z_{2}$, где $Z_{1}$ - конечный веер, а $Z_{2}$ бесконечен и все подгруппы из $Z_{2}$ являются группами $\Phi$ робениуса с неинвариантньм множителем, содержащим элемент $a$. При этом ядра любых двух подгрупп из $Z_{2}$ пересекаются тривиально. Ввиду лемм 1,4 элемент $a$ не перестановочен ни с одной инволюцией групшы $G$. Учитывая предложение 1 , приходим к вьводу, что содержащие элемент $a$ дополнения всех фробениусовых подгрупп из $Z_{2}$ суть некоторые подгруппы из $R=N_{G}(\langle a\rangle)$. Нетрудно убедиться, что свойство тривиальности попарных пересечений ядер подгрупп из $Z_{2}$ можно 
значительно усилить, а именно: $Z_{2}=Z_{0} \cup Z_{3}$, где $Z_{0}$ - пустой или конечный веер, а ядро любой подгрупшы $H$ из $Z_{3}$ не имеет общих неединичных элементов с любой подгруппй $M \in Z$, отличной от $H$.

Ввиду леммы $4 R=N_{G}(\langle a\rangle)$ - конечная подгруппа. Учитьвая вышесказанное, приходим к выводу, что только для конечного числа элементов $c=r c_{a} \in N_{b}$ элементы $c_{a}$ не содержатся в подгруппах из веера $Z_{3}$. Поэтому, не ограничивая общности рассуждений, будем считать, что уже все элементы вида $c_{a}$ содержатся в подгруппах из $Z_{3}$ и, следовательно, в ядрах этих фробениусовых подгрупш. Пусть $H$ - подгруппа из $Z_{3}$, содержащая элемент $c_{a}$, и $F$ - ее ядро. Из свойств веера $Z_{3}$ вытекает, что подгрупшы $L^{+}$ и $L^{-}$содержатся в $H$. Действительно, $L^{+}$содержится в некоторой максимальной подгруппе $M$, и так как $a \in L^{+}$, то $M \in Z$. Поскольку $c_{a} \in F \cap L^{+}$, то $M=H$ и $L^{+} \leqslant H$. Аналогично, $L^{-} \leqslant H$.

Итак, $b c, b^{-1} c \in H, b^{2}=b c\left(b^{-1} c\right)^{-1} \in H$, и так как порядок элемента $b$ нечетен (лемма 5), то $b \in H$ и $c \in H$. Поскольку $a b=b a$ (лемма 8), то элемент $b$ содержится в неинвариантном множителе группы $H$. Отсюда заключаем, что $c \in F$ и $r=c c_{a}^{-1} \in F$. Но $C_{F}(a)=1$; следовательно, $r=1$ и $c=c_{a} \in N_{a}$. Таким образом, множество $N_{b} \backslash N_{a}$ не более чем конечно, и лемма доказана.

На бесконечных подмножествах групшы $G$ введем отношение $\sim: X \sim Y$ в том и только том случае, если $|X \backslash Y|<\infty$ и $|Y \backslash X|<\infty$. Нетрудно убедиться, что есть отношение эквивалентности.

ЛЕмма 10. Имеем $N_{a} \sim N_{b}$.

ДоКАЗАТЕЛЬСТво. Ввиду леммы 6 рассматриваемая нами ситуация симметрична относительно элементов $a$ и $b$. Так, поменяв местами элементы $a, b$ и повторив рассуждения лемм 8,9 , докажем, что множество $N_{a} \backslash N_{b}$ конечно. С учетом леммы 9 это означает, что $N_{a} \sim N_{b}$. Лемма доказана.

По лемме $4 C_{G}(a)$ - конечная группа и, следовательно, она содержит конечное число $n$ различных циклических подгрупп $\left\langle b_{1}\right\rangle, \ldots,\left\langle b_{n}\right\rangle$, сопряженных в $G$ с подгруппой $\langle b\rangle$.

Лемма 11. Подгруппа $B=\left\langle b_{1}, b_{2}, \ldots, b_{n}\right\rangle$ ииклическая, т.е. $n=1$.

ДокАЗАТЕЛЬСтво. Снова рассмотрим веер $X_{a}$ конечных подгрупш $L_{g}=\left\langle a, b^{g}\right\rangle$, где $g \in G$. Ввиду леммы $8 X_{a}=Y \cup X$, где $Y$ - конечный веер, а веер $X$ бесконечен и состоит из фробениусовьх подгрупп с циклическими дополнениями порядка $p q$. Поскольку в качестве элемента $b$ мы можем выбрать любой элемент из элементов $b_{1}, \ldots, b_{n}$, то в соответствии с леммой 8 (и теоремой 7) получаем разбиение веера $X=X_{1} \cup \cdots \cup X_{n}$ на бесконечные вееры $X_{1}, \ldots, X_{n}$ с основаниями $\left\langle a b_{i}\right\rangle$. Применяя лемму 10, получаем

$$
N_{a} \sim N_{b_{1}}, \ldots, \quad N_{a} \sim N_{b_{n}}, \quad N_{b_{i}} \sim N_{b_{j}}, \quad 1 \leqslant i, j \leqslant n .
$$

Отсюда выводим, что для множества $N=N_{a} \cap N_{b_{1}} \cap \cdots \cap N_{b_{n}}$ выполняется $N \sim N_{a}$ и множество $N_{a} \backslash N$ конечно.

Пусть $Z$ - веер всех максимальных по включению фробениусовых подгрупп из $G$ с неинвариантным множителем $\langle a\rangle$. Ввиду теоремы $7 Z=Z_{1} \cup Z_{2}$, где $Z_{1}$ - конечный веер, $Z_{2}$ - бесконечный веер и ядро каждой подгруппы $H \in Z_{2}$ имеет тривиальное пересечение с ядром любой отличной от $H$ подгрупшы из $Z_{2}$. Очевидно, что все элементы из $N$ содержатся в ядрах подгрупп из $Z$. Выбросив из $Z_{2}$ подгруппы, ядра которых имеют неединичные пересечения хотя бы с одной из подгрупп веера $Z_{1}$, мы получим веер $Z_{3}$, 
ядра подгрупп $H$ которого имеют единичное пересечение с любой подгруппой $M \in Z$, отличной от $H$. Нетрудно убедиться, что веер $Z \backslash Z_{3}$ конечен.

Обозначим через $N_{0}$ множество элементов из ядер подгрупп веера $Z_{3}$. Ввиду вьшедоказанного $N_{0} \sim N$.

Пусть $c$-произвольный элемент из $N_{0}$ и $H_{c}$ - подгрупа из $Z_{3}$, содержащая элемент $c$, a $F_{c}$ - ее ядро. Поскольку $N_{0} \subseteq N_{a} \cap N_{b_{i}}(1 \leqslant i \leqslant n)$, то подгруппы $\left\langle b_{i} c\right\rangle$ сопряжены в $G$ с подгруппой $\langle b\rangle$ и $L_{c}=\left\langle a, b_{i} c\right\rangle$ - конечная группа $\Phi$ робениуса с циклическим неинвариантным множителем порядка $p q$. По свойствам конечных групा Фробениуса элемент $c$ содержится в подгруппе $L_{c}$ (предложение 1 ). Значит, $L_{c}-$ группа Фробениуса с неинвариантным множителем $\left\langle a b_{i}\right\rangle$ и ядром $R_{c}$, содержащим элемент $c$. В силу свойств множества $Z_{3}$ заключаем, что $R_{c} \leqslant F_{c}$ и $b_{i} \in N_{G}\left(F_{c}\right)(i=1, \ldots, n)$.

Пусть $C$ - подгруппа, порожденная элементами $a, b_{1}, \ldots, b_{n}$. Как доказано вьше, подгруппа $C$ нормализует каждую подгруппу $F_{c}$, где $c \in N_{0}$. Поэтому каждая из подгрупп $F_{c} \lambda C=K_{c}$, где $c \in N_{0}$, конечна и они составляют бесконечный веер $W$ с основанием $D$, содержащим подгруппу $C$. Ввиду теоремы 7 почти все подгруппы $K_{c} \in W$ являются группами $\Phi$ робениуса с неинвариантньм множителем $C$ и ядром $F_{c}$. Используя конечность подгруппы $C_{G}(a)$, утверждение 3$)$ леммы 1 и предложение 1 , заключаем, что $C$ не содержит инволюций. Теперь из свойств неинвариантного множителя в конечной группе Фробениуса (предложение 1 ) вытекает, что $B=\left\langle b_{1}, \ldots, b_{n}\right\rangle \leqslant K_{c}-$ циклическая группа простого порядка $q$. Поэтому $B=\langle b\rangle$ и $n=1$. Лемма доказана.

Завершим доказательство теоремы 9. Применяя леммы 10, 11, заключаем, что почти все элементы класса $b^{G}$ содержатся в множестве $\langle b\rangle \cdot N_{0}$, где $N_{0}=N_{a} \cap N_{b}$. Понятно, что для любого элемента $c \in N_{0}$ подгруппа $\langle b, c\rangle$ конечна. Поэтому почти для всех элементов $b^{g}$ подгруппы $\left\langle b, b^{g}\right\rangle$ конечны и являются группами $\Phi$ робениуса с неинвариантньп множителем $\langle b\rangle$. Поскольку $\left|b^{G}\right|=\infty$, то отсюда и из предложения 2 следует равенство $G=F \lambda N_{G}(\langle b\rangle)$, что противоречит простоте группы $G$. Полученное противоречие означает, что теорема 9 верна.

ДОКАЗАТЕЛЬСТВО ТЕОРЕМЫ 10. 1) Пусть выполняются условия утверждения 1) теоремы, и предположим, что $L=\langle a, c\rangle \neq G$ для некоторого элемента $c \in b^{G}$. Тогда $L$ - конечная группа и ввиду теоремы 4 и условий утверждения $1 \neq L \cap H \neq L \cap M \neq 1$. По теореме $5 L$ - группа Фробениуса и $L \cap H, L \cap M-$ ее дополнения. Но тогда по предложению $1 L \cap H=L \cap M^{x}$ для некоторого элемента $x \in L$ и $H \cap M^{x} \neq 1$ вопреки теореме 4 и условиям утверждения. Следовательно, $G=\langle a, c\rangle$ для любого элемента $c \in b^{G}$.

2) Пусть $L_{c}=\langle a, c\rangle \neq G$ для бесконечного числа элементов $c \in b^{G}$. Тогда эти подгруппы $L_{c}$ являются конечными группами фробениуса с дополнениями $L \cap M=\langle c\rangle$ (теорема 5), а ввиду предложения 1 их ядра $F_{c}$ попарно различны и нильпотентны. Но тогда по лемме 2 централизатор $C_{G}(a)$ бесконечен, противоречие. Значит, доказываемое утверждение верно.

3) Снова допустим, что $L_{c}=\langle a, c\rangle \neq G$ для бесконечного числа элементов $c \in b^{G}$. Тогда ввиду предложения 6 конечные подгрупшы $L_{c}$ почти все являются группами $\Phi$ робениуса с дополнениями, содержащими элемент $a$. Применяя предложение 1 , заключаем, что подгруппы $\langle a\rangle$ и $\langle c\rangle$ сопряжены в $L_{c}$ вопреки условиям. Утверждение доказано.

4) Предполагая, что доказьваемое утверждение неверно, рассмотрим бесконечньй веер $X$ конечных подгрупп $L_{c}=\langle a, c\rangle \neq G$, где $c \in b^{G}$. Поскольку любая собствен- 
ная подгрупша $K$ в $G$ слойно конечна, то она может включать в себя не более чем конечное число подгрупп из $X$. Учитьвая предложение 6 , заключаем, что почти каждая подгруппа $L_{c}$ из $X$ есть группа $ф$ робениуса с дополнением $T_{c}$, содержащим элемент $a$. Из предложения 1 , леммы 1 и конечности $C_{G}(a)$ следует, что порядок $T_{c}$ нечетен. Поэтому если элемент $c$ не лежит в ядре фробениусовой группы $L_{c}$, то по предложению 1 $b^{G} \cap C_{G}(a)$ не пусто вопреки условиям утверждения. Следовательно, каждьй элемент $c$ из фробениусовой группы $L_{c}$ содержится в ее ядре. Но тогда получаем противоречие с леммой 3. Итак, утверждение верно.

5) Если $K=\langle b, c\rangle$, то $i \in N_{G}(K)$ и ввиду теорем 6,5 и предложения $1 K \lambda\langle i\rangle-$ группа Фробениуса с абелевым ядром $K$. Согласно теореме 5 это может выполняться только для конечного числа элементов $c \in N_{a}$.

Теорема доказана.

\section{СПИСОК ЦИТИРОВАННОЙ ЛИТЕРАТУРЫ}

[1] Черников С.Н. К теории бесконечных $p$-групп // Докл. АН СССР. 1945. Т. 50. № 1. C. $71-74$.

[2] Черников С.Н. Бесконечные слойно конечные группы // Матем. сб. 1948. Т. 22. № 64. C. 101-133.

[3] Черников С. Н. Группы с заданными свойствами системы подгрупп. М.: Наука, 1980.

[4] Горчаков Ю. М. Группы с конечными классами сопряженных элементов. М.: Наука, 1978.

[5] Сенашов В. И. Слойно конечные группы. Новосибирск: ВО Наука, 1993.

[6] Созутов А.И. О существовании в группе $f$-локальных подгрупп // Алгебра и логика. 1997. Т. 36. № 5. C. 573-598.

[7] Созутов А.И., Шахова С.И. О квазислойно конечных группах // Вест. Крас. архит.-строит. акад. Вып. 1. Сб. науч. тр. Красноярск, 1999. С. 77-83.

[8] Созутов А.И., Шахова С. И. О строении квазислойно конечных групп // Вест. Крас. архит.-строит. акад. Вып. 1. Сб. науч. тр. Красноярск, 2000. С. 69-76.

[9] Ольшанский А. Ю. Геометрия определяющих соотношений в группах. М.: Наука, 1989.

[10] Шунков В.П. О периодических группах с почти регулярной инволюцией // Алгебра и логика. 1972. Т. 11. № 4. С. 470-493.

[11] Шунков В.П. О достаточных признаках существования в группе бесконечных локально конечных подгрупп // Алгебра и логика. 1976. Т. 15. №6. С. 716-737.

[12] Лоссов К.И. Достаточные условия вложимости амальгамы в периодическую группу // Тезисы сообщений 19-й Всесоюзной алгебраической конференции. Ч. 1. Львов, 1987. С. 163.

[13] Коуровская тетрадь: Нерешенные вопросы теории групп. Изд-е 14-е. Новосибирск, 1999.

[14] Бусаркин В. М., Горчаков Ю. М. Конечные расщепляемые группы. М.: Наука, 1968.

[15] Старостин А. И. О группах Фробениуса // Укр. матем. ж. 1971. Т. 23. № 5. С. 629-639.

[16] Созутов А. И., Шунков В. П. Об одном обобщении теоремы Фробениуса на бесконечные группы // Матем. сб. 1976. Т. 100. № 4. С. 495-506.

[17] Созутов А.И., Шунков В. П. О бесконечных группах, насыщенных фробениусовыми подгруппами // Алгебра и логика. 1977. Т. 16. №6. С. 711-735.

[18] Созутов А.И. О некоторых признаках непростоты групп с инволюциями // Алгебра и логика. (в печати).

[19] Каргаполов М.И., Мерзляков Ю. И. Основы теории групп. М.: Наука, 1977.

[20] Харари Ф. Теория графов. М.: Мир, 1973.

Красноярская государственная архитектурно-строительная академия

Поступило

E-mail: root@sozutov.krasnoyarsk.su

28.08.2000

Исправленньй вариант

11.07.2001 